\title{
Proinflammatory chemokine gene expression influences survival of patients with non-Hodgkin's lymphoma
}

\author{
Grzegorz Mazur $^{1}$, Emilia Jaskuła ${ }^{2}$, Ilona Kryczek², Dorota Dłubek ${ }^{2}$, \\ Aleksandra Butrym ${ }^{1}$, Tomasz Wróbel ${ }^{1}$, Andrzej Lange ${ }^{2}$, Kazimierz Kuliczkowski ${ }^{1}$ \\ ${ }^{1}$ Department of Hematology, Blood Neoplasms and Bone Marrow Transplantation, \\ Wroclaw Medical University, Poland \\ ${ }^{2}$ Department of Clinical Immunology, L. Hirszfeld Institute of Immunology and Experimental \\ Therapy, Polish Academy of Sciences, Wroclaw, Poland
}

\begin{abstract}
The migration, survival and proliferation of cells is the basis for all physiologic and pathologic processes in the human body. All these reactions are regulated by a complex chemokine network that guides lymphocytes homing, chemotaxis, adhesion and interplay between immunologic system response cells. Chemokines are also responsible for metastatic dissemination of cancers, including Hodgkin's and non-Hodgkin's lymphomas. The purpose of this study was to determine chemokine gene expression (CXCL8, CXCL10, CCL2, CCL3, CCL4 and CCL5) in lymphoma lymph nodes compared to their expression in reactive lymph nodes. We also analyzed the influence of chemokine gene expression on the survival of lymphoma patients. Chemokine gene expression was evaluated in 37 lymphoma lymph nodes and in 25 samples of reactive lymph nodes. Gene expression of chemokines CXCL8, CXCL10, CCL2, CCL3, CCL4 and CCL5 was measured using the PCR method. Statistical analysis was performed using CSS Statistica for Windows (version 7.0) software. Probability values < $<0.05$ were considered statistically significant and those between 0.05 and 0.1 as indicative of a trend. We found lower CXCL8 and CXCL10 gene expression in lymphoma lymph nodes compared to reactive lymph nodes. In the cases of CCL2 and CCL3, expression in lymphomas was higher than in reactive lymph nodes. Patients with high expression of CCL2 and CXCL10 had shorter survival. (Folia Histochemica et Cytobiologica 2011; Vol. 49, No. 2, pp. 240-247)
\end{abstract}

Key words: lymphoma, chemokines, gene expression

\section{Introduction}

Chemokines are small proteins with low molecular mass, which display a pleiotropic action and are secreted mainly by leukocytes and some other tissue cells. The chemokine family is divided into four subgroups, based on conservative cysteine residues in polypeptide chain. Chemokines act through specific

Correspondence address: G. Mazur, Department of Hematology, Blood Neoplasms and Bone Marrow Transplantation,

Pasteur Str. 4, 50-367 Wroclaw, Poland; tel.: (+ 48 71) 78425 99, fax: (+ 48 71) 78401 12;

e-mail: grzegorzmaz@yahoo.com transmembrane G-protein-coupled receptors [1]. Chemokines play an important role in the homeostatic process, particularly in regulating the migration and homing of leukocytes in peripheral lymphatic organs $[2,3]$. On the other hand, it is known that chemokines take part in many pathological processes, such as inflammation, the neoplastic process, and cancer progression and dissemination $[4,5]$.

Non-Hodgkin's lymphoma (nHL) comprises a heterogenous group of lymphatic system neoplastic disorders. Lymphomas derive from lymphocytes B, $\mathrm{T}$ or NK or their precursors. It has been suggested that chemokines, as factors regulating immunocompetent cells migration and activity, are responsible for 
the disruption of immunologic processes and could have an important role in lymphomagenesis. Lymphomas can localize not only to lymph nodes, but disseminate into different extranodal organs. Chemokines also play an important role in the migration of lymphoma cells [6].

Hodgkin's lymphoma (HL) is a particular type of cancer in which the tumor mass contains reactive cells (such as lymphocytes, plasmocytes, eosinophils, histiocytes, fibroblasts) and a clonal population of B-cells unable to secrete immunoglobulins (RSC, Reed-Sternberg cells) [7]. RSC comprise about $1 \%$ of all tumor cells. It has been suggested that infiltration of a tumor by reactive cells is caused by RSC chemokine secretion and their chemotactic activity on reactive cells. Higher expression of chemokines CXCL9, CXCL10, CCL5, and CCL3 has been demonstrated in tissues infiltrated by HL [8], but expression of each of these chemokines has been linked to a different cell population [9]. The previous theory suggested the domination of Th2 immunologic response in HL [10]. The latest studies have shown a predomination of regulatory $\mathrm{T}$ cells rather than Th2 lymphocytes [11] and explained why neoplastic cells can in this case avoid antineoplastic immunologic control.

NHL and HL are two different groups of diseases in both a histopathological and a clinical sense. The involvement of lymph nodes occurs in practically all cases, but nHL has a stronger predisposition for extranodal localization than HL. As a process that always accompanies cancer, inflammation joins both types of lymphoma. Tumor cells secrete chemokines and cytokines, attracting inflammation cells, which also can promote tumor growth and progression [12]. On the other hand, lymphadenopathy can be present in both lymphoma and inflammation. Lymph node metastases and clinical reactive lymph node enlargement differs in the profile of chemokines and their receptors expressed [13].

The purpose of this study was to evaluate proinflammatory chemokine gene expression (CXCL8, CXCL10, CCL2, CCL3, CCL4 and CCL5) in lymphoma lymph nodes compared to their expression in reactive lymph nodes. We also analyzed the influence of chemokine gene expression on the survival of lymphoma patients.

\section{Material and methods}

Lymph nodes. Chemokine gene expression was evaluated in 37 lymphoma lymph nodes taken from patients (16 women, 21 men, aged 18-81 years; median age 43) at the time of diagnosis. It was also evaluated in 25 samples of reactive lymph nodes (taken from 15 women and 10 men aged 18-59; median age 32) (Table 1).
Table 1. Patient characteristics

\begin{tabular}{|l|c|c|c|}
\hline $\begin{array}{c}\text { Lymphoma } \\
\text { type }\end{array}$ & $\begin{array}{c}\text { No. of } \\
\text { patients }\end{array}$ & $\begin{array}{c}\text { Sex } \\
\text { F/M }\end{array}$ & $\begin{array}{c}\text { Median age } \\
\text { (range) years }\end{array}$ \\
\hline B-cell lymphoma & 26 & $12 / 14$ & $57(26-73)$ \\
FL & 7 & & \\
MCL & 4 & & \\
SLL & 4 & & \\
DLBCL & 4 & & \\
MZL & 3 & & \\
BL & 2 & & \\
MM & 1 & & \\
HCL & 1 & & $59(41-81)$ \\
\hline T-cell lymphoma & 4 & $0 / 4$ & \\
PTCL & 1 & & $27(21-58)$ \\
ALCL & 3 & & \\
\hline Hodgkin's & 7 & $4 / 3$ & \\
lymphoma & & & \\
\hline
\end{tabular}

FL — follicular lymphoma; MCL — mantle cell lymphoma;

SLL — small lymphocytic lymphoma; DLBCL — diffuse large B-cell lymphoma; MZL — marginal zone lymphoma; BL — Burkitt lymphoma; MM - multiple myeloma; HCL — hairy cell leukemia; PTCL — peripheral T-cell lymphoma; ALCL — anaplastic large cell lymphoma

Methods. Samples of the studied lymphomas (37) and reactive lymph node tissues (25) were divided into two parts. One was fixed in $10 \%$ buffered formalin and then embedded in paraffin. Sections were stained with hematoxylin and eosin and evaluated histopathologically. The second parts of the lymph nodes, used for gene expression analysis, were snap frozen in liquid nitrogen and stored at $-70^{\circ} \mathrm{C}$.

Chemokine gene expression analysis. Total RNA was extracted from the frozen tissue samples using Trizol reagent (Invitrogen Corp., Carlsbad, CA, USA). The amount of isolated total RNA was estimated quantitatively by spectrophotometric measurement and qualitatively by electrophoretical view. First-strand cDNA was synthesized using First-Strand Synthesis System (Stratagene, La Jolla, CA, USA) according to the manufacturer's specifications. cDNA used for PCR was standardized toward to $\beta$-actin mRNA. Briefly, $\beta$-actin was initially amplified and quantified with serial dilutions of cDNA from each sample. CXCL8, CXCL10, CCL2, CCL3, CCL4 and CCL5 were then amplified in each sample containing identical amounts of $\beta$-actin mRNA. Gene expression was estimated in arbitrary units (AU) using a 0 to 3 point AU scale. PCR was conducted using primer pairs for CCL2 (sense TCC AGC ATG AAA GTC TCT GC, antisense TGG AAT CCT GAA CCC ACT TC, 245 bp); CCL3 (sense GTC ATC TTC CTA ACC AAG CG, antisense TGT GGC TGT TTG GCA ACA AC, 229 bp); CCL4 (sense AGG AAG CTT CCT CGC AAC TT, antisense AGT CCT GAG TAT GGA GGA GA, 244 bp); CCL5 (sense CAT TGC TAC TGC CCT CTG CG, antisense GGG TTG GCA CAC ACT GTT CG, 192 bp); CXCL8 (sense TTG GCA GCC TTC CTG ATT, antisense 
AAC TTC TCC ACA ACC CTC TG, 403 bp); CXLC10 (sense TGG CAT TCA AGG AGT ACC TC, antisense TGT AGG GAA GTG ATG GGA GA, 326 bp); and $\beta$-actin (sense GGG TCA GAA GGA TTC CTA TG, antisense GGT CTC AAA CAT GAT CTG GG, 250 bp).

Amplification was performed in a MJ Research PTC-200 Peltier thermal cycler DNA engine (MJ Research Inc, Watertown, MA, USA). The PCR conditions were 35 cycles at $95^{\circ} \mathrm{C}$ for 30 seconds, $55^{\circ} \mathrm{C}$ (for $\beta$-actin, CXCL10), or $60^{\circ} \mathrm{C}$ (for CCL2-5), or $68^{\circ} \mathrm{C}$ (for CXCL8) for 30 seconds, and $72^{\circ} \mathrm{C}$ for 30 seconds, followed by final extension at $72^{\circ} \mathrm{C}$ for ten minutes.

The size of PCR products was determined by agarose gel electrophoresis.

Statistical analyses. Univariate analyses were performed using Fisher's exact test or $\chi^{2}$ test. Cumulative survival was analyzed via Kaplan-Meier plots. Probability values $<0.05$ were considered statistically significant and those between 0.05 and 0.1 as indicative of a trend. Statistical analysis was performed using Statistica for Windows (version 7.0) software (Sta-Soft Inc., Tulsa, OK, USA).

\section{Results}

\section{CXCL8 gene expression}

In most cases, CXCL8 gene expression was low, or was not detected at all. In four samples: two HL, one T-cell lymphoma and one reactive node, CXCL8 expression was increased ( 2 or $3 \mathrm{AU}$ ). In $62 \%$ of cases of B-cell lymphoma, CXCL8 expression was not shown. Only FL (five cases) and SLL (four cases) revealed gene expression at the level of 1 AU. In $50 \%$ of reactive lymph nodes, CXCL8 expression was at $1 \mathrm{AU}$ level. In other reactive lymph nodes, expression was not detected. In three T-cell lymphomas, there was low (1 AU) expression of the CXCL8 gene. In one case, expression was detected at a 3 AU level.

\section{CXCL10 gene expression}

CXCL10 expression was studied in 62 lymph nodes. In reactive lymph nodes, expression was high in 18/ $25(72 \%)$ cases. All lymphoma lymph nodes expressed CXCL10 at a lower (1 AU) level ( $\mathrm{p}=0.002)$. In all T-cell lymphomas, expression at a level of 2 AU was observed. In five cases of HL, expression of chemokine was at a low (1 AU) level. Two other cases did not express CXCL10. In B-cell lymphomas, the level of CXCL10 expression was statistically lower than in reactive lymph nodes $(\mathrm{p}=0.002)$. In these patients, a higher expression (2 AU) was detected in DLBCL, BL and MCL. In lymphoma patients, we found a correlation between overall survival and CXCL10 expres-

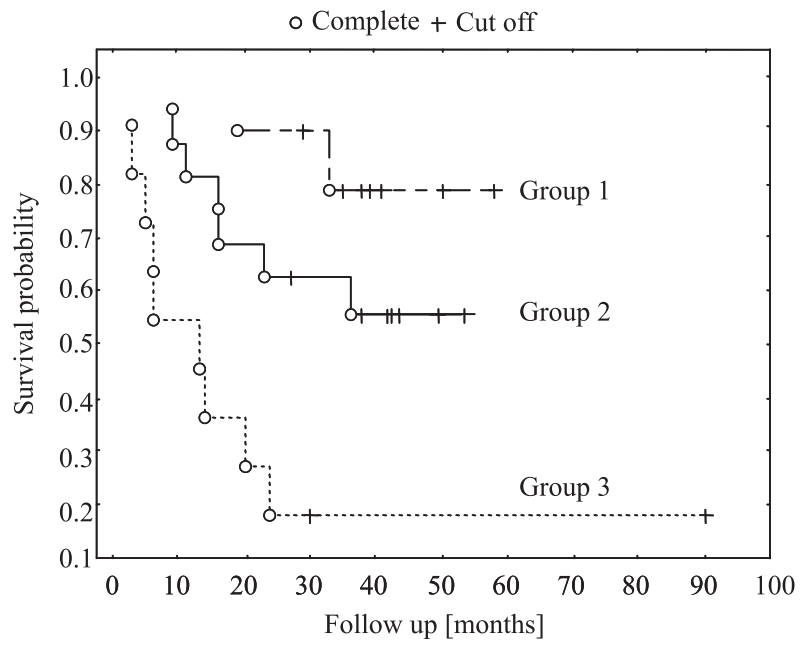

Figure 1. Kaplan-Meier cumulative survival plots comparing lymphoma patients with different CXCL10 expressions: Group 1: 2-3 AU CXCL10 expression; Group 2: 1 AU CXCL10 expression; Group 3: 0 AU CXCL10 expression

sion. Patients with higher CXCL10 expression had statistically shorter survival than those with low CXCL10 expression ( $\mathrm{p}=0.002)$ (Figure 1).

\section{CCL2 gene expression}

Analysis of lymphoma lymph nodes revealed a statistically higher level of CCL2 expression compared to reactive lymph nodes ( $\mathrm{p}=0.0008)$. In 19/26 (73\%) of B-cell lymphomas, and all T-cell lymphomas, a 2-3 AU expression was observed. In 4/7 (57\%) cases of HL, expression of CCL2 was detected. Expression of CCL2 was present in 7/25 (28\%) of reactive lymph nodes, but was statistically lower than in B-cell lymphomas $(\mathrm{p}=0.001)$. Particularly high CCL2 expression was characteristic for DLBCL and Burkitt lymphoma. In FL, MCL and SLL, expression was comparable at around 1-2 AU. In multiple myeloma and hairy cell leukemia, the expression of CCL2 was not detected. In lymphoma patients, we found a correlation between overall survival and CCL2 expression. Patients with higher CCL2 expression had statistically shorter survival than those with low CCL2 expression $(\mathrm{p}=0.004)$.

\section{CCL3 gene expression}

Almost all $(33 / 37 ; 89 \%)$ lymphoma lymph nodes showed a CCL3 expression of 2 AU. In reactive lymph nodes, expression was more differentiated (0 to $3 \mathrm{AU}$ ). Analysis of the total group of lymphoma lymph nodes revealed statistically higher CCL3 gene expression ( $\geq 2 \mathrm{AU})$ than in reactive lymph nodes $(9 / 25 ; 36 \%)$, $\mathrm{p}=0.01$. Low expression was observed in only four lymphoma lymph nodes: one HL, two FL and one MZL. 


\section{CCL4 gene expression}

Expression of CCL4 was detected in 58/62 (94\%) analyzed lymph nodes. In most cases expression was at a high ( $\geq 2 \mathrm{AU})$ level. There was no statistically significant difference between lymphoma and reactive lymph nodes.

\section{CCL5 gene expression}

CCL5 gene expression was observed in 60/62 (97\%) lymph nodes (no expression in one MM and one reactive lymph node). All lymphoma types revealed higher, but comparable, CCL5 expression level (in $30 \%$ of cases, expression equaled $3 \mathrm{AU}$ ). In almost all (except one) HL lymph nodes, expression was equal to 2 AU. In T-cell lymphomas, expression varied between 1 and 3 AU. In B-cell lymphoma, a particularly high expression was found in BL and 50\% of MCL (Figures 2 and 3).

\section{Discussion}

Chemokines are small proteins $(812 \mathrm{kD})$ which bind to specific receptors on target cells, and can influence proliferation, migration, apoptosis and activation of normal and neoplastic cells [14-17]. Research is revealing their role in inflammation, immunologic reactions, organ formation and organization, and carcinogenesis [18].

In our study we analyzed proinflammatory chemokine gene expression in lymphomas (HL and $\mathrm{nHL}$ ) and reactive lymph nodes. The state of inflammation very often accompanies neoplasms and seems to be a promoting factor for cancer development. Neoplastic cells can secrete chemokines, which in an autocrine manner stimulate their growth (for example: in melanoma CXCL1, CXCL2, CXCL3, CXCL4 [19]), simultaneously causing recruitment of inflammatory cells. A special connection between inflammation and cancer can be observed in HL, in which infiltrating reactive cells secrete an increased amount of chemokines (mainly of the CC group) [9]. Neoplastic and reactive lymphadenopathy can be differentiated by histopathologic examination of the lymph node. The migration capacity of cells towards lymph nodes depends on chemokines and receptors expression, but, importantly, one receptor can bind chemokines of different affinities. Corcione et al. showed migration of MCL cells in vitro in response to CCL19 and CXCL12 [19].

CXCL8 can be produced by leukocytes (monocytes, T lymphocytes, neutrophils, NK cells) and also fibroblasts and epithelial cells. Its expression can be induced by proinflammatory cytokines (interleukin 1 ) and tumor necrosis factor- $\alpha(\mathrm{TNF} \alpha)$. CXCL8 is responsible mainly for leukocyte migration to peripheral tissues, and also for degranulation of neutrophils. In our study we showed low expression of CXCL8. In both B-cell lymphomas and reactive lymph nodes, expression was almost undetectable. CXCL8 shows an activity for freshly-isolated T lymphocytes, but incubation of lymphocytes causes a progressive drop of receptors binding CXCL8 on $\mathrm{T}$ lymphocytes and is combined with decreased immunologic response [20]. CXCL8 indirectly can induce $\mathrm{T}$ lymphocytes chemotaxis through release of chemoattractants from neutrophils and is also chemotactic for B cells [21]. Fujii et al. studied the expression of chemokines in DLBCL with different localizations (nodal, extranodal, central nervous system involvement) [22]. In general, expression was low, but higher in patients with nodal localization. Another study revealed CXCL8 expression in HL-stimulated lines and in the serum of patients with HL [23]. Research in situ did not show either the presence of CXCL8 transcript nor protein in RSC, but CXCL8 was present in reactive neutrophils in HL [24]. Those results are in line with ours. It is known that neutrophils play an important role in neoplastic infiltration, because they attract inflammation cells and sustain the inflammation process.

CXCL10 chemokine is a key mediator of response to interferon. CXCL10 attracts to the site of inflammation Th1 activated lymphocytes, and also can inhibit angiogenesis [25]. CXCL10 expression occurs in activated mononuclear cells, keratinocytes, fibroblasts, endothelial cells and T lymphocytes. In vitro CXCL10 inhibits colony formation by human hematopoietic cells in bone marrow [26] and shows strong anti-neoplastic activity in vivo [27]. CXCL10 is chemotactic for monocytes and $\mathrm{T}$ lymphocytes and can promote $\mathrm{T}$ cell adhesion to the endothelium [28].

We found high CXCL10 expression level in $72 \%$ of studied lymph nodes. All lymphoma lymph nodes had significantly lower CXCL10 expression. Maggio et al., using the RT-PCR method, demonstrated CXCL10 expression in HL cell lines, but not in NHL cell lines [29]. CXCL10 is a marker of immunologic reactions and its expression is stimulated by proinflammatory factors. Before CXCL10 was found to be a chemotactic substation, it had been used to determine interferon-gamma (INF $\gamma$ ) levels. Our results might suggest a decreased grade of polarization towards Th2 population in the inflammation process. More probably, it is a process with general proinflammatory stimulation, with both Th1 and Th2 lymphocyte recruitment. 


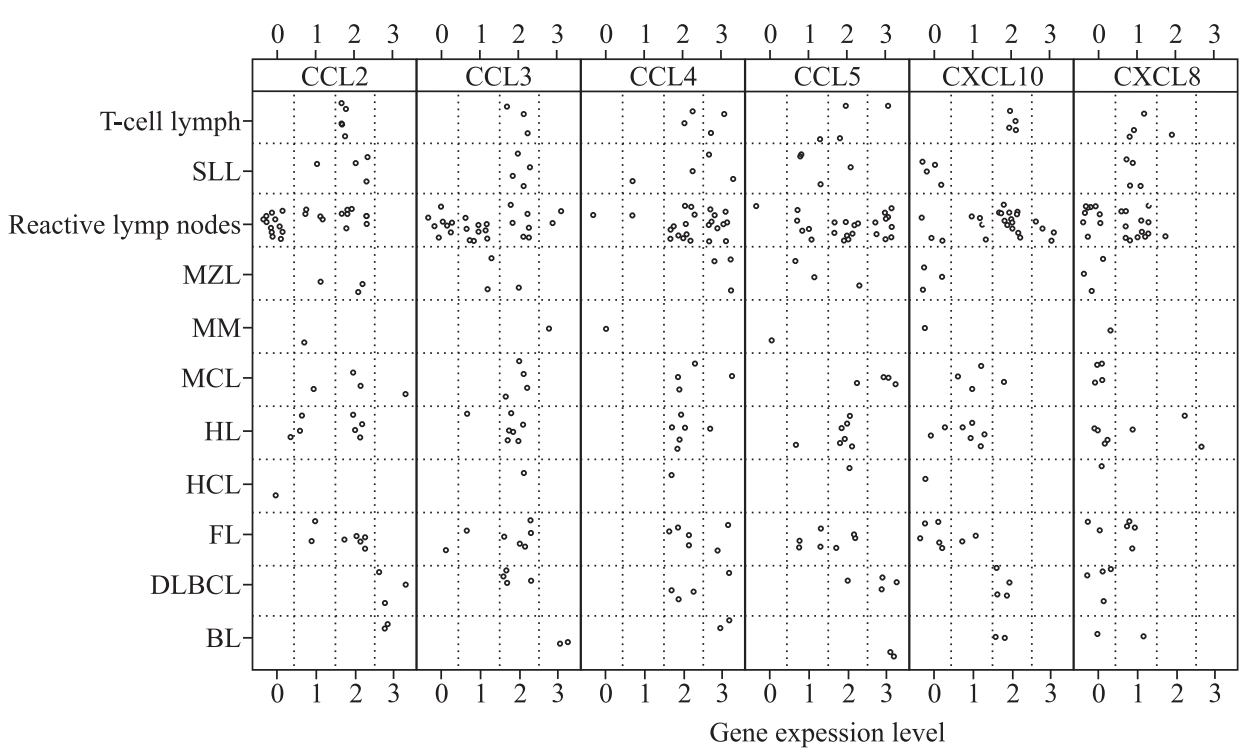

Figure 2. Chemokine gene expressions in different types of lymphomas and reactive lymph nodes

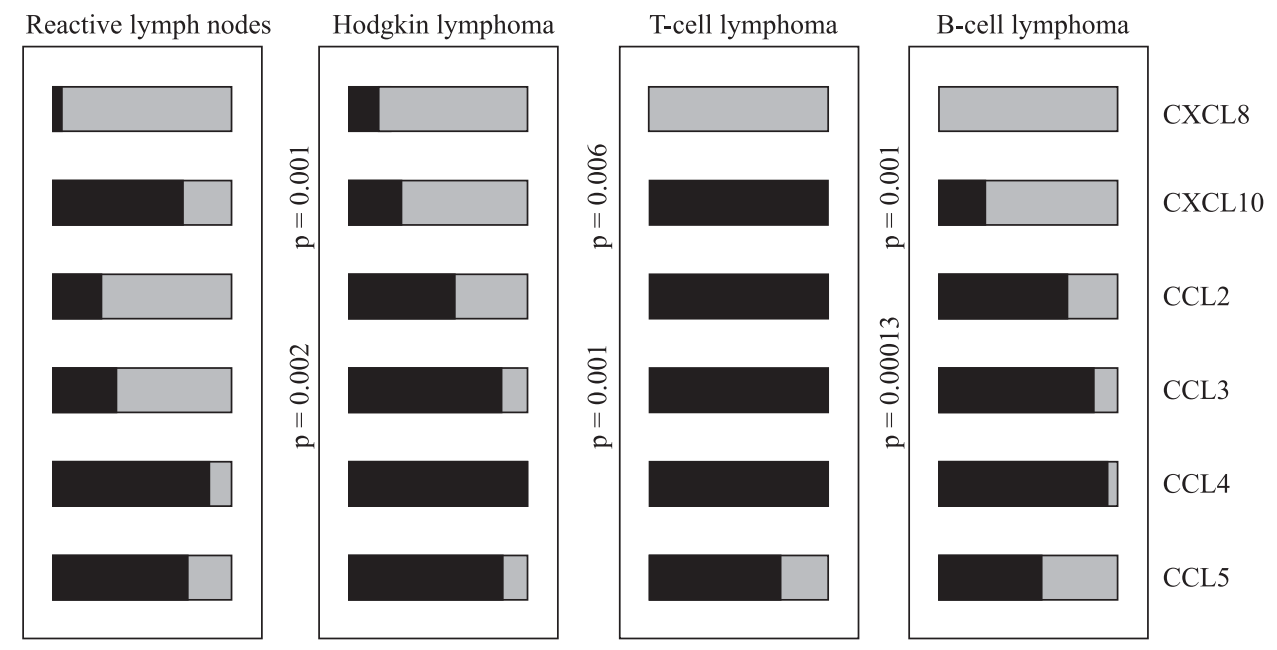

Figure 3. Comparison of proportional gene expression in subgroups of B- and T-cell lymphomas, Hodgkin's lymphoma and reactive lymph nodes. Gray — the number of lymph nodes with low expression ( $0,1 \mathrm{AU})$; black — the number of lymph nodes with high expression (2, 3 AU). 'p' values represent comparison between special lymphoma subgroup and reactive lymph nodes

We also noticed an important correlation between CXCL10 expression and patient survival: increased expression was connected to shorter survival. Grade of CXCL10 expression as marker protein of INF $\gamma$ reflects potential Th1 response. Teichmann et al., analyzing CXCL10 expression in HL, came to a similar conclusion [30]. INF $\gamma$ alone can be a strong anti-cancer factor. Additionally activated by INF $\gamma$ cytotoxic lymphocytes and NK cells could play a role in anti-neoplastic control. Thus it is surprising that a negative correlation between CXCL10 expression and clinical prognosis was found in our study. CXCL10 alone has angiostatic and apoptotic activity, and should limit cancer progression.
CCL2 induces chemotaxis of monocytes lymphocytes T and NK, dendritic cells and basophils. CCL2 can be secreted not only by tumor cells but also by stromal cells such as fibroblasts, endothelial cells and monocytes. CCL2 inhibits tumor growth through activity of T lymphocytes. But on the other hand, it promotes cancer progression (tumor-associated macrophages recruitment and proangiogenic activity). Recently, we demonstrated increased mean CCL2 plasma levels in untreated patients with acute myeloid leukemia [31]. The role of CCL2 in nHL is yet to be established. Lucciani et al. showed CCL2 expression in 23/24 HL, 7/17 B-cell nHL and 13/25 cancers deriving from lungs, breast, thyroid and ovary [23]. 
An increased level of CCL2 could be linked to a higher proinflammatory cytokine expression. For example, interleukin- 6 and TNF $\alpha$ are responsible for "B" symptoms occurrence. Husson et al. noticed that in follicular lymphoma, an increase in CCL2 secretion is secondary to higher $\mathrm{TNF} \alpha$ production, and that chemotaxis of neoplastic cells can be modulated by CCL2 [32].

In our study, CCL2 expression in lymphoma lymph nodes was significantly higher than in reactive lymph nodes. This is probably linked to increased angiogenesis in the lymphoproliferative process. Monitoring of chemokines with proangiogenic activity could have prognostic value. Our present study found high CCL2 expression to result in shorter survival, which can have a prognostic impact in nHL. This finding can have therapeutic implication with use of angiogenesis inhibitors in clinical practice.

CCL3 is a chemokine with an important role in multiple myeloma [33, 34]. CCL3 stimulates differentiation, increases osteolytic activity and attracts osteoclasts. There has been shown to be a correlation between increased CCL3 level and grade of osteolytic features and stage of diseases in multiple myeloma patients $[34,35]$. We observed higher CCL3 expression in lymphoma lymph nodes compared to reactive nodes. The role of CCL3 in nHL has not been described yet. Matsuhashi et al. presented a case report of a patient with diffuse B-cell lymphoma, who demonstrated strong osteolytic changes in bones at the diagnosis of lymphoma [36]. Additionally increased CCL3 expression was present, which could explain massive osteolysis. In our study, lymphoma lymph nodes were characterized by higher CCL3 expression compared to the reactive ones. In the aforementioned work of Fujii et al., in all cases of DLBCL, CCL3 expression was detected and increased in lymphomas with central nervous system involvement [22].

The CCL4 chemokine, which binds to CCR5 receptor, is related particularly to T lymphocytes. This chemokine is involved in antigen-dependent B-cell activation [36]. In our study, we did not notice differences in CCL4 expression between lymphoma and reactive lymph nodes. It is known that CCL4 attracts $\mathrm{CD}^{+} / \mathrm{CDRO}^{+} \mathrm{T}$-cells [36] and activated B-cells in mice show chemotactic activity on regulatory $\mathrm{T}$ lymphocytes through CCL4 [37, 38]. Recent work by Ek et al. concerning CCL4 expression in MCL showed CCL4 expression in lymphoma tissues as well as in MCL cell lines [39]. In the study performed by Fujii, in DLBCL expression of CCL4 was observed and the level was particularly increased in nodal localization of DLBCL [22]. Little is known about CCL4's role in lymphomas. Normal B-cells, after receptor rearrangement, can secrete CCL4 chemokine. It is probable that a high expression of CCL4, which is involved in T-cell regulation, can influence the growth and survival of cancer cells in nHL.

CCL5, acting through CCR1, CCR3 and CCR5 receptors, is chemotactic for monocytes, T lymphocytes, eosinophils and mastocytes [40]. In T-cell population, CCL5 generally influences memory cells with Th1 phenotype. CCL 5 secreted by CD $8{ }^{+}$lymphocytes, thrombocytes, epithelial cells and fibroblasts, and recruits leukocytes to the site of inflammation. Its expression has been shown in some neoplasms: Hodgkin's lymphoma (either CD20 cells or RSC), breast cancer [41], prostate cancer [42] and melanoma [43], in which expression level was correlated with decreased anti-neoplastic response and cancer progression. In our study, we observed high CCL5 expression in the majority of examined lymph nodes, without significant differences between lymphoma subgroups. In the study by Ek et al. on lymphoma tissues and MCL cell lines, high CCL5 expression was also observed [39].

Our results suggest that high expression of CCL5 (chemokine regulating $\mathrm{T}$ cells and follicular dendritic cells) in nHL can promote tumor growth. We also found a positive correlation between CCL5 expression and proliferation index Ki-67 in lymphomas. Murooka et al. found a new role for CCL5 as an important regulator of T-cell activity through apoptosis induction [44]. At high concentration, CCL5 activates mitochondrial/apoptosome pathway, induces apoptosis and causes T lymphocytes depletion, determining immunologic response. Buri et al. noticed higher expression of CCR 3 and CCR5 receptors for CCL5 on B cells isolated from lymph nodes infiltrated by Hodgkin's lymphoma [9]. Interestingly, in the case of B lymphocytes isolated from reactive and $\mathrm{nHL}$ lymph nodes, a similar situation did not occur. Recently, it has been shown that CCL5 as a ligand for CCR5 is possibly involved in regulation of HL and RSC growth and the formation of a microenvironment typical for Hodgkin's lymphoma [45].

The studies of inflammation and cancer have always run in parallel. Virchow in 1863 observed the presence of lymphocytes in tumor tissue [46]. Inflammation sustains the proliferation of cancer cells by stromal cells activation and chemokines secretion. Some chemokines, such as CXCL12 and CXCL13, have proven roles in the pathogenesis of lymphoma $[47,48]$.

In our study, we observed different chemokine gene expressions in lymphoma lymph nodes compared to reactive lymph nodes. Lymphomas were characterized by higher CCL2 and CCL3 expression, and lower CXCL8 and CXCL10 expression than in reactive lymph nodes. The expressions of CCL2 and 
CXCL10 seem to be important in cases of nHL, as they have been shown to have value in predicting patients' survival. Growing understanding of the mechanisms that regulate cancer cell migration and proliferation will help in introducing target therapy, which will replace or supplement standard chemotherapy.

\section{References}

1. Horuk R. Chemokine receptors. Cytokine Growth Factor Rev. 2001;4:313-335.

2. Paoletti S, Petkovic V, Sebastiani S et al. A rich chemokine environment strongly enhances leukocyte migration and activities. Blood. 2005;105:3405-3412.

3. de Gorter DJ, Beuling EA, Kersseboom R et al. Bruton's tyrosine kinase and phospholipase Cgamma2 mediate chemokine-controlled B cell migration and homing. Immunity. 2007;26:93-104.

4. Kakinuma T, Hwang ST. Chemokines, chemokine receptors and cancer metastasis. J Leukocyt Biol. 2006;79:639-651.

5. Zlotnik A, Yoshie O. Chemokines: a new classification system and their role in immunity. Immunity. 2000;12:121-127.

6. Laurence AD. Location, movement and survival: the role of chemokines in hematopoiesis and malignancy. Br J Haematol. 2006;132:255-267.

7. Marafioti T, Hummel M, Foss HD et al. Hodgkin and Reed-Sternberg cells represent an expansion of a single clone originating from a germinal center B-cell with functional immunoglobulin gene rearrangements but defective immunoglobulin transcription. Blood. 2000;95:1443-1450.

8. Maggio E, van den Berg A, Diepstra A et al. Chemokines, cytokines and their receptors in Hodgkin's lymphoma cell lines and tissues. Ann Oncol. 2002;13:(Suppl 1):52-56.

9. Buri C, Körner M, Schärli P et al. CC chemokines and the receptors CCR3 and CCR5 are differentially expressed in the non-neoplastic leukocytic infiltrates of Hodgkin disease. Blood. 2001;97:1543-1548.

10. Poppema S, van den Berg A. Interaction between host T cells and Reed-Sternberg cells in Hodgkin lymphomas. Semin Cancer Biol. 2000;10:345-350.

11. Marshall NA, Christie LE, Munro LR et al. Immunosuppressive regulatory $\mathrm{T}$ cells are abundant in the reactive lymphocytes of Hodgkin lymphoma. Blood. 2004;103:1755-1762.

12. Moore MA. The role of chemoattraction in cancer metastases. Bioassays. 2001;23: 674-676.

13. Lopez-Giral S, Quintana NE, Cabrerizo M et al. Chemokine receptors that mediate $\mathrm{B}$ cell homing to secondary lymphoid tissues are highly expressed in B-cell chronic lymphocytic leukemia and non-Hodgkin lymphomas with widespread nodular dissemination. J Leukoc Biol. 2004;76:1-9.

14. Moldobaeva A, Baek A, Eldridge L et al. Differential activity of pro-angiogenic CXC chemokines. Microvasc Res. 2010; doi.org/10.1016/j.mvr.2010.01.011.

15. Mazur G, Jaskuła E, Kryczek I. Udział chemokin w chorobach nowotworowych. Adv Clin Exp Med. 2004;13:315-325.

16. Kim $\mathrm{CH}$. The greater chemotactic network for lymphocyte trafficking: chemokines and beyond. Curr Opin Hematol. 2005;12:298-304.

17. Złotnik A. Chemokines in cancer progression. Semin Cancer Biol. 2004;14:181-185.

18. Trentin L, Cabrelle A, Facco M et al. Homeostatic chemokines drive migration of malignant $\mathrm{B}$ cells in patients with non-Hodgkin lymphomas. Blood. 2004;104:502-508.
19. Corcione A, Arduino N, Ferretti E et al. CCL19 and CXCL12 trigger in vitro chemotaxis of human mantle cell lymphoma B cells. Clin Cancer Res. 2004;10:964-971.

20. Hess C, Means TK, Autissier P et al. IL-8 responsiveness defines a subset of CD8 T cells poised to kill. Blood. 2004;104:3463-3471.

21. Jinquan T, Moller B, Storgaard M et al. Chemotaxis and IL-8 receptor expression in B cells from normal and HIV-infected subjects. J Immunol. 1997;158:475-484.

22. Fujii A, Ohshima K, Hamasaki M et al. Differential expression of chemokines, chemokine receptors, cytokines and cytokine receptors in diffuse large B cell malignant lymphoma. Int J Oncol. 2004;24:529-538.

23. Luciani MG, Stoppacciaro A, Peri G et al. The monocyte chemotactic protein 1 (MCP-1) and interleukin 8 (IL-8) in Hodgkin disease and solid tumors. J Clin Pathol. 1998; 51:273-276.

24. Foss HD, Herbst $\mathrm{H}$, Gottstein $\mathrm{S}$ et al. Interleukin-8 in Hodgkin's disease: preferential expression by reactive cells and association with neutrophil density. Am J Pathol. 1996;148:1229-1236.

25. Hancock WW, Gao W, Csizmadia V et al. Donor-derived IP-10 initiates development of acute allograft rejection. J Exp Med. 2001;193:975-980.

26. Sarris AH, Broxmeyer HE, Wirthmueller U et al. Human interferon-inducible protein 10: expression and purification of recombinant protein demonstrate inhibition of early human hematopoietic progenitors. $J$ Exp. 1993;178:1127-1132.

27. Luster AD, Leder P. IP-10, a -C-X-C- chemokine, elicits a potent thymus-dependent antitumor response in vivo. $J$ Exp Med. 1993;178:1057-1065.

28. Taub DD, Lloyd AR, Conlon K et al. Recombinant human interferon-inducible protein 10 is a chemoattractant for human monocytes and $\mathrm{T}$ lymphocytes and promotes $\mathrm{T}$ cell adhesion to endothelial cells. J Exp Med. 1993;177:1809-1814 .

29. Maggio EM, van den Berg A, Visser L. Common and differential chemokine expression patterns in rs cells of NLP, EBV positive and negative classical Hodgkin lymphomas. Int J Cancer. 2002;99:665-672.

30. Teichmann M, Meyer B, Beck A et al. Expression of the interferon-inducible chemokine IP-10 (CXCL10), a chemokine with proposed anti-neoplastic functions, in Hodgkin lymphoma and nasopharyngeal carcinoma. J Patho. 2005; 22206:68-75.

31. Mazur G, Wróbel T, Butrym A et al. Increased monocyte chemoattractant protein 1 (MCP-1/CCL-2) serum level in acute myeloid leukemia. Neoplasma. 2007;54:285-289.

32. Husson H, Carideo EG, Cardoso AA et al. MCP-1 modulates chemotaxis by follicular lymphoma cells. Br J Haematol. 2001;15:554-562.

33. Giuliani N, Colla S, Rizzoli V. New insight into the mechanism of osteoclast activation and formation in multiple myeloma: focus on the receptor activator of NF-kappaB ligand (RANKL). Exp Hematol. 2004;32:685-691.

34. Roodman GD, Choi SJ. Mip-1 alpha and myeloma bone disease. Cancer Treat Res. 2004;118:83-100.

35. Pals ST, de Gorter DJ, Spaargaren M. Lymphoma dissemination: the other face of lymphocyte homing. Blood. 2007;110:3102-3111.

36. Matsuhashi Y, Tasaka T, Uehara E et al. Diffuse large B-cell lymphoma presenting with hypercalcemia and multiple osteolysis. Leuk. Lymphoma. 2004;45:397-400. 
37. Bystry RS, Aluvihare V, Welch KA et al. B cells and professional APCs recruit regulatory T cells via CCL4. Nat Immunol. 2001;2:1126-1132.

38. Taub DD, Conlon K, Lloyd AR et al. Preferential migration of activated $\mathrm{CD}_{4}^{+}$and $\mathrm{CD} 8{ }^{+} \mathrm{T}$ cells in response to MIP-1 alpha and MIP-1 beta. Science. 1993;260:355-358.

39. Ek S, Björck E, Högerkorp CM et al. Mantle cell lymphomas acquire increased expression of CCL4, CCL5 and 4-1BB-L implicated in cell survival. Int $J$ Cancer. 2006; 118:2092-2097.

40. Appay V, Rowland-Jones SL. RANTES: a versatile and controversial chemokine. Trends Immunol. 2001;22:83-87.

41. Azenshtein E, Luboshits G, Shina S et al. The CC chemokine RANTES in breast carcinoma progression. Regulation of expression and potential mechanism of promalignant activity. Cancer Res. 2002;62:1093-1102.

42. Peehl DM, Kadam PA, Lawrence DM. Expression of CCL5 (RANTES) and CCR5 in prostate cancer. Prostate. 2006; 66:124-134.
43. Payne AS, Cornelius LA. The role of chemokines in melanoma tumor growth and metastasis. J Invest Dermatol. 2002;118:915-922.

44. Murooka TT, Wong MM, Rahbar R et al. CCL5-CCR5 mediated apoptosis in T cells: requirement for glycosaminoglycan binding and CCL5 aggregation. J Biol Chem. 2006; 281:25184-25194.

45. Aldinucci D, Lorenzon D, Cattaruzza Let al. Expression of CCR5 receptors on Reed-Sternberg cells and Hodgkin lymphoma cell lines: involvement of CCL5/Rantes in tumor cell growth and microenvironmental interactions. Int J Cancer. 2008;122:769-776.

46. Virchow R. Die Krankhaften Geschwulste. Hirschwald, Berlin, 1863.

47. Fischer L, Korfel A, Pfeiffer S et al. CXCL13 and CXCL12 in central nervous system lymphoma patients. Clin Cancer Res. 2009;15:5968-5973.

48. de Oliveira KB, Oda JM, Voltarelli JC et al. CXCL12 rs1801157 polymorphism in patients with breast cancer, Hodgkin's lymphoma, and non-Hodgkin's lymphoma. J Clin Lab Anal. 2009;23:387-393.

Submitted: 29 June, 2010

Accepted after reviews: 30 November, 2011 